\title{
How should we diagnose and manage photosensitivity?
}

\author{
S Ibbotson \\ Photobiology Unit, Ninewells Hospital and Medical School, Dundee
}

KEYWORDS photosensitivity, phototesting, polymorphic light eruption, sunlight, sunscreen, ultraviolet radiation

DECLARATION OF INTERESTS No conflict of interests declared.

\author{
Correspondence to S Ibbotson \\ Photobiology Unit \\ Dermatology Department \\ Ninewells Hospital and Medical \\ School \\ Dundee DDI 9SY \\ UK
}

When we expose ourselves to natural sunlight this is mainly visible light; ultraviolet radiation (UVR) is only a small component of natural daylight (Figure I). Of the UVR, most will be UVA with less than $5 \%$ being UVB. Ultraviolet exposure will depend on the time of the year. For example in northern Europe we will not receive any UVB exposure in winter and relatively less UVA on sunny winter days than we would be exposed to in the summer.

The term 'photosensitivity' is used to describe the acute reaction of skin to UVR. This may fall within normal population responses and individuals may be categorised according to their sensitivity to sunburn and their ability to tan using the Fitzpatrick skin phototype classification.' Photosensitivity is considered abnormal if the reaction of skin to UVR is either quantitatively or qualitatively outwith that of the normal population. Thus, if an individual has a lowered susceptibility to developing a sunburn-like reaction after minimal sun exposure, or develops a rash after sun exposure, this would be considered to be abnormal.

Photosensitivity diseases are a heterogeneous group of conditions (Table I) and detailed clinical assessment is vital in order to elicit a history of photosensitivity and to provide clues to the characteristics of the reaction and thus the possible diagnosis. The age of onset of the condition is important as there are some disorders such as actinic prurigo, hydroa vacciniforme and erythropoietic protoporphyria which tend to present in childhood and others, such as chronic actinic dermatitis, which usually occur in later life. It is essential to determine the characteristics of the type of ultraviolet or visible light exposure which is required to trigger the abnormal photosensitivity. Thus, establishing whether the condition is triggered throughout the year or just in spring and

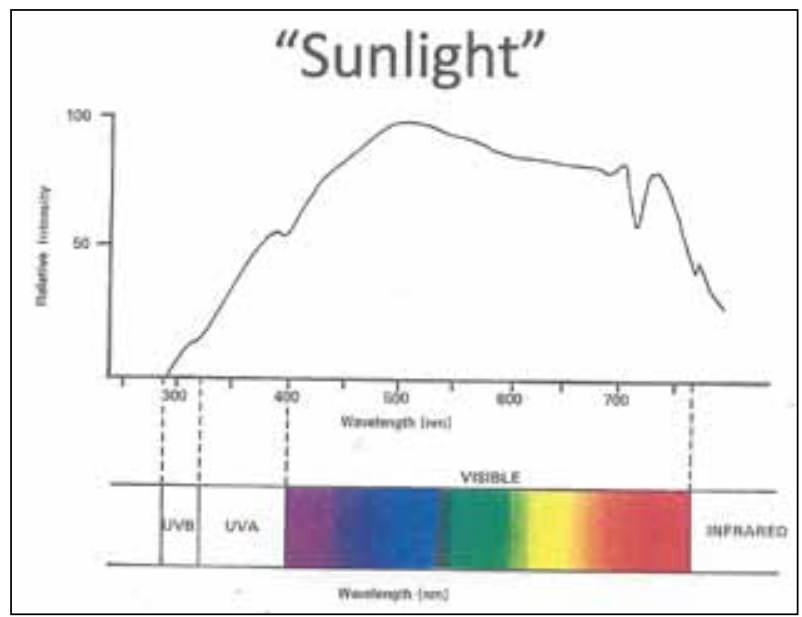

FIGURE I Emission spectrum of solar exposure demonstrating the relative proportions of UVR and visible light. From Frain-Bell W. Cutaneous Photobiology. Oxford University Press: Oxford; 1985. p. 136-9

summer and whether it is only direct sunlight exposure, window glass transmitted light and/or sunbed exposure that will trigger the problem are key factors. Disorders in which UVA and visible light photosensitivity feature are more likely to be associated with perennial symptoms as opposed to disorders restricted particularly to UVB and the shorter UVA wavelengths which will often be more common in spring and summer. Window glass absorbs UVB and allows transmission of UVA therefore if photosensitivity is triggered with light through window glass this implies that UVA and/or visible wavelengths are triggering the problem.

It is also important to establish the time required to produce the photosensitivity reaction and the lag period 


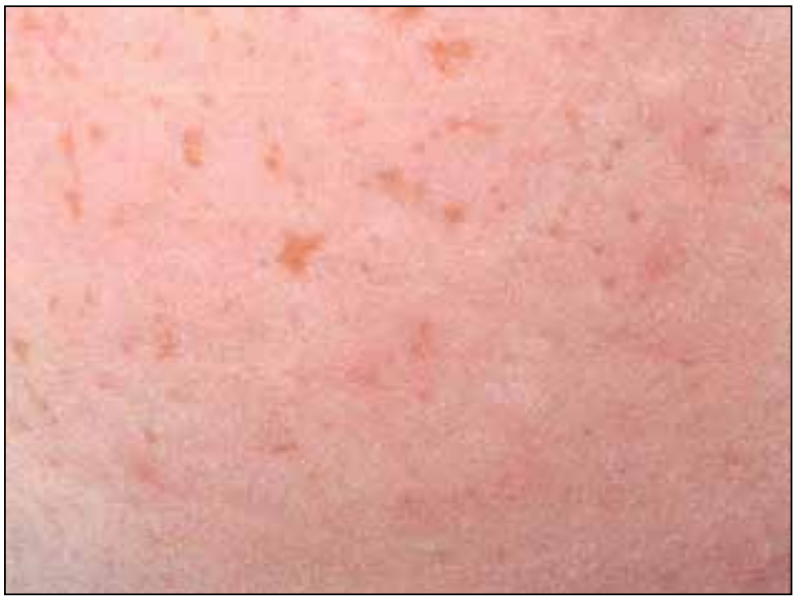

FIGURE 2 Subtle vacciniforme scarring on the cheek of a child with hydroa vacciniforme

until this occurs. For example, polymorphic light eruption typically requires a few hours of exposure to trigger an itchy rash which may not develop until later that day or the following day and will last for a few days before resolution. In contrast, solar urticaria will usually be triggered within minutes of exposure at any time of the year and lesions will resolve within an hour or two. If clothing is not protective and the patient still develops the rash through lightly woven clothing then this implies that UVA and/or visible light are implicated in triggering the photosensitivity. The presence of hardening (reduced sensitivity on habitually exposed sites such as face and back of hands), is much more common, for example in polymorphic light eruption, than in some of the other photosensitive conditions, such as solar urticaria or chronic actinic dermatitis.

Symptoms are informative and anyone with skin pain on sun exposure should have porphyria excluded as this raises suspicion of a cutaneous porphyria. Itching is usually more prevalent in many of the other disorders, such as polymorphic light eruption. A fundamental part of history taking is a detailed drug history as many drugs may cause phototoxicity through non-immunological phototoxic mechanisms. These include antibiotics (e.g. fluoroquinolones and doxycycline), diuretics (e.g. thiazides), non-steroidal anti-inflammatory drugs (oral and topical), quinine, phenothiazines, calcium antagonists, amiodarone or ingested St John's wort. It is essential to pinpoint this from the history as (if this is the diagnosis) once the culprit drug is stopped the photosensitivity should resolve. Family history may be relevant in some of the photosensitive conditions such as in actinic prurigo, with $50 \%$ of Caucasian patients having a positive family history for the disease, rising to $75 \%$ in South America. It is important to discuss and obtain details of the patient's occupation as this may well be relevant, for example in the case of photocontact allergy to specific photoallergens such as occupational non-steroidal antiinflammatory drug exposure. ${ }^{2}$ Likewise, recreational $^{2}$

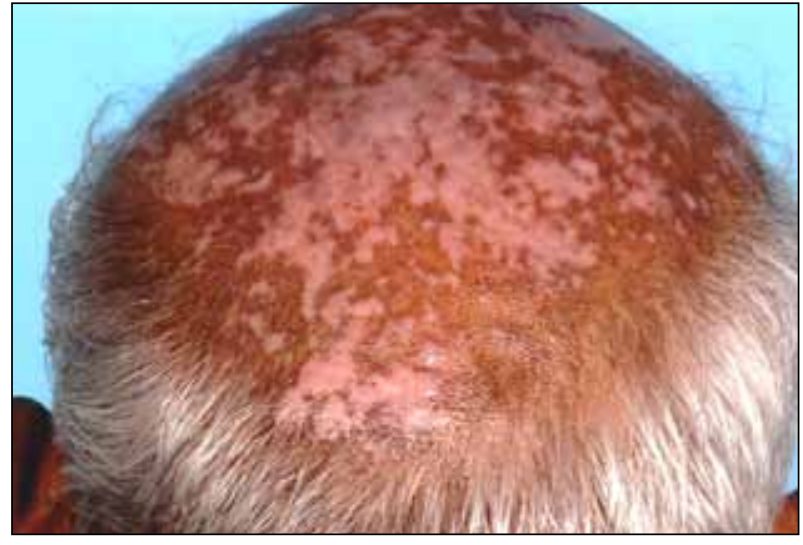

FIGURE 3 Leucomelanoderma arising in a patient with skin phototype $V$ with chronic actinic dermatitis

TABLE I Classification of the photodermatoses Idiopathic

Polymorphic light eruption

Chronic actinic dermatitis

Solar urticaria

Actinic prurigo

Juvenile spring eruption

Hydroa vacciniforme

\section{Drugs and chemicals}

Exogenous: systemic and topical

Endogenous: porphyrias

\section{Photogenodermatoses}

Xeroderma pigmentosum

Rothmund Thomson, Bloom syndrome and others

\section{Photoaggravated}

exposure may be important as in the case of phytophotodermatitis or 'strimmer's' dermatitis. There is emerging evidence ${ }^{3-6}$ to demonstrate that chronic and, in particular severe photosensitivity, has a significant adverse impact on quality of life. This is important to be aware of and relatively high levels of depression and anxiety are reported in this group of patients.

On examination, there are usually clues to the presence of a photodistributed rash, with prominent involvement of photoexposed sites on the head and neck including the forehead, nose, chin, sternocleidomastoid and mastoid areas, balding head, top of ears and back of neck. Typically these may be sparing around the eyes and in the shadows cast by the ears (Wilkinson's triangle), sideburns, lower lip and under the chin. Characteristically, in airborne contact dermatitis, the upper eyelids are involved and in photosensitivity these are spared. Likewise with airborne contact dermatitis, the back of the neck is typically spared whereas in photosensitivity this is a commonly affected site. The extensor surfaces of arms and forearms and back of hands may be most prominently affected and on the lower limbs photosensitivity will typically affect the extensor surfaces, particularly the tops of thighs and tops of feet. 


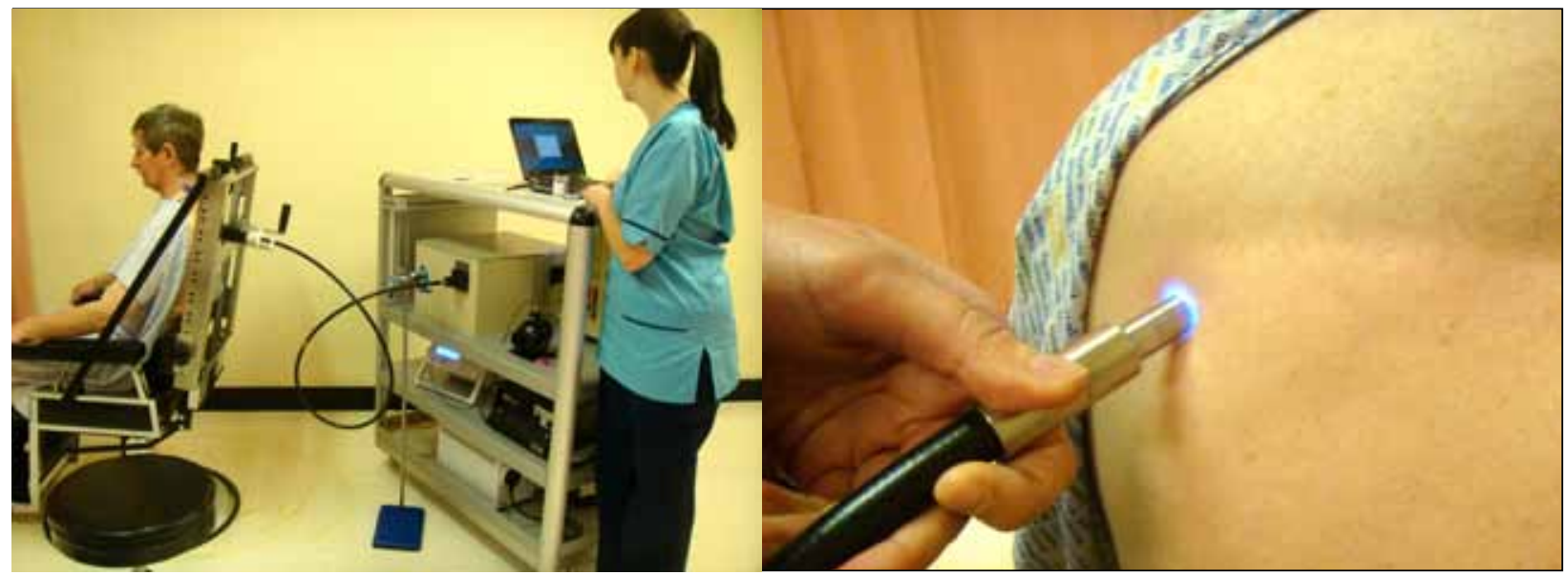

FIGURE 4 Monochromator phototesting. (Left) Phototesting with patient sitting comfortably. Filtered xenon arc source (on trolley) is coupled to a fibre-optic light guide. (Right) Irradiation undertaken at test sites on clinically normal appearing back skin and delivered via fibre-optic light guide

Ascertaining the nature of a rash is important: papules and vesicles would lead to consideration of, for example, polymorphic light eruption or actinic prurigo, or if vesicle fluid is haemorrhagic then hydroa vacciniforme. Vacciniforme scarring, which may be subtle (Figure 2), should be looked for as it does make a diagnosis of hydroa vacciniforme more likely. If the rash is urticated in nature then this is suggestive of either primary or secondary solar urticaria, and if there is erythema and later desquamation then the possibility of drug phototoxicity should be considered. The presence of photoexposed site dermatitis should lead to consideration of a diagnosis of chronic actinic dermatitis, photocontact allergic dermatitis or the main differential diagnosis of airborne contact allergic dermatitis. Pigmentary changes such as leucomelanoderma commonly occur with certain drug photosensitisers such as quinine or thiazides but can also occur in chronic actinic dermatitis (Figure 3). Skin fragility, scarring and milia should always alert suspicions of a cutaneous porphyria.

If a patient is suspected to have a photosensitivity disorder then they should be referred for investigation to a specialist photodiagnostic unit. The objectives of investigation are to define the nature of the photosensitivity and establish a firm diagnosis (Table 2). The mainstay investigation is monochromator phototesting which is the use of a xenon arc source which is filtered to allow delivery of narrow wavebands of light across the solar spectrum in order to establish erythemal responses and compare these with a normal population range (Figure 4). The minimal erythema dose (MED) is the threshold dose of irradiation required to cause just perceptible erythema. This is the objective endpoint usually measured at 24 hours after monochromator phototesting and is used to determine most cases of abnormal photosensitivity. In solar urticaria where immediate photosensitivity is the problem, the

\section{TABLE 2 Objectives of investigation}

- To establish if the patient is abnormally photosensitive

- If so, to determine the action spectrum and degree of abnormal photosensitivity

- To determine a firm diagnosis

\section{TABLE 3 Investigations that may be used in a patient with suspected photosensitivity \\ - Monochromator phototesting \\ - Minimal erythema dose (MED) determination \\ - $\quad$ Provocation testing \\ - Phototesting to compact fluorescent lamps \\ - Patch testing \\ - $\quad$ Photopatch testing \\ - $\quad$ ANA, ENAs \\ - Porphyrin plasma scan \\ - HLA typing \\ - $\quad$ Skin biopsy}

minimal urticarial dose (MUD), the dose of irradiation required to elicit just detectable urticaria, is used as the main outcome measure. Other investigations will often include broadband provocation testing usually with UVA and patch or photopatch testing. The latter is undertaken to sunscreens and topical non-steroidal antiinflammatories and is used to investigate possible photocontact allergy to these drugs and chemicals. ${ }^{7}$ Screening for lupus, the cutaneous porphyrias or other rarer photosensitivity diseases may be required (Table 3 ).

With the introduction of environmentally friendly low energy compact fluorescent lighting, these sources now emit more into the UV part of the spectrum and can pose a risk for severely photosensitive patients such as those with solar urticaria, chronic actinic dermatitis or lupus. ${ }^{8-10}$ It is now possible to undertake phototesting 


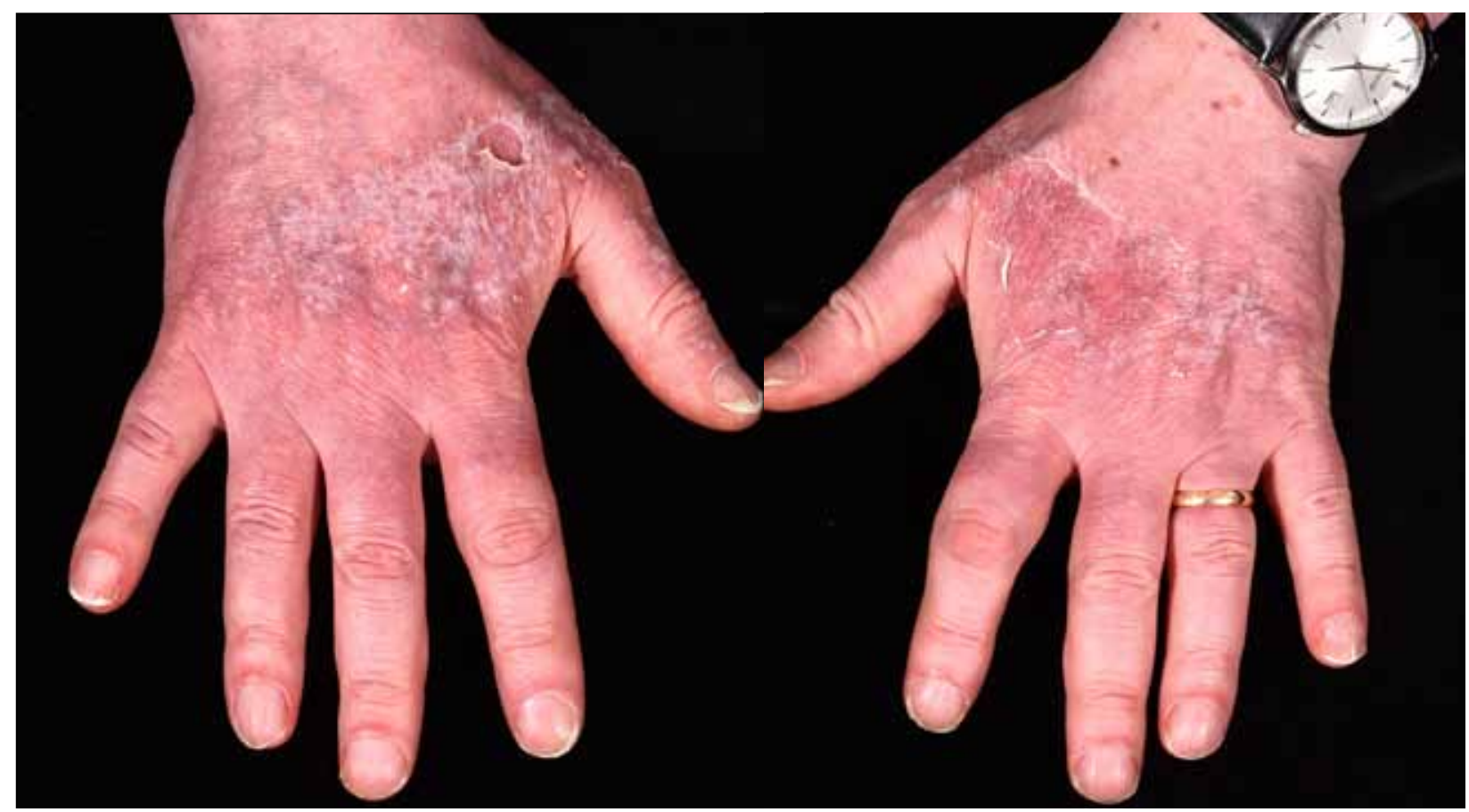

FIGURE 5 Vemurafenib phototoxicity. Note prominent involvement of photo-exposed sites on back of hands. Sunlight (UVA) exposure a few days earlier resulted in a blistering phototoxic reaction

with these compact fluorescent light sources and this can be helpful in providing information and advice for patients regarding lighting choices, notably the use of double enveloped compact fluorescent lamps or LEDs (Table 3). MED testing to lamps that can be used to treat photosensitive disorders, through induction of desensitisation and tolerance, are also often undertaken and MED testing is an essential safety measure in order to pick up any abnormal photosensitivity reactions before a treatment course of phototherapy (Table 3). Patients referred for investigation often have many investigations done and it is important to look at the clinical relevance of any investigation findings. Furthermore, investigations may be negative and yet the patient may still have exacerbation of their condition by light such as in the photoaggravated skin diseases, for example rosacea or seborrhoeic dermatitis.

Idiopathic photodermatoses include the majority of abnormal cutaneous reactions to sunlight (Table I) and this is explained on the basis of the prevalence of polymorphic light eruption in 18\% of European populations." A further category of abnormal photosensitivity diseases is those induced by drugs and chemicals. These include endogenous chemicals in the porphyrias, exogenous systemic or topical drugs and chemicals (for example with systemic quinine, thiazides, fluoroquinolones, doxycycline or amiodarone phototoxicity) or topical photocontact allergy to nonsteroidal anti-inflammatories applied directly to the skin or sunscreen chemicals. Some of the more recently introduced drugs, such as the BRAF inhibitor vemurafenib, used for metastatic melanoma (Figure 5),

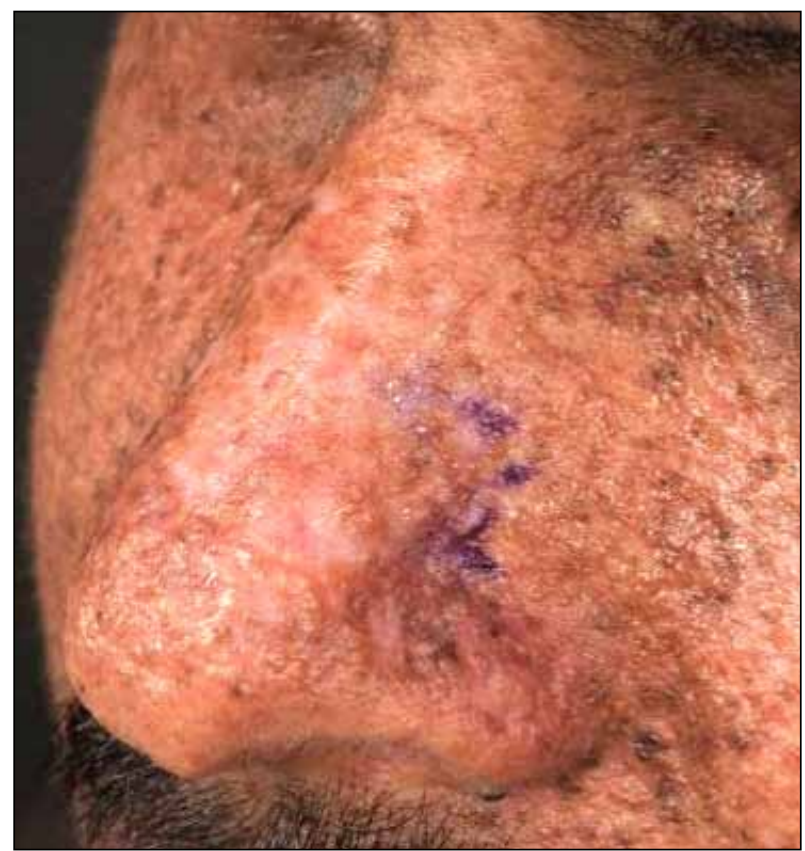

FIGURE 6 Abnormal pigmentary change (lentigines and hypopigmented macules), dysplastic changes and scarring in a patient of skin phototype $\mathrm{V}$ with xeroderma pigmentosum. The purple marker pen demonstrates a site to be biopsied. (c/o Dr Hiva Fassihi, Clinical Lead for the National XP Service, Guy's \& St Thomas' Hospital, London)

voriconazole, pirfenidone for lung fibrosis and antihepatitis $C$ agents have also been associated with photosensitivity. Most drug photosensitivity is mediated by UVA and visible wavelengths. 
Photogenodermatoses are a rare group of diseases in which photosensitivity is a manifestation and key aspect of the disease. The best known is xeroderma pigmentosum (Figure 6) which is a rare group of autosomal recessive conditions in which DNA excision repair pathways or post replication repair pathways are abnormal. Patients develop markedly accelerated photoaging and nonmelanoma skin cancers and many will also have severe delayed abnormal photosensitivity. Photoaggravated photodermatoses constitute another relatively large group of conditions which are exacerbated but not caused by light and it may well be that the infra-red component of sunlight is part of the trigger.

The key points to stress are that it is important to have a low threshold for thinking about photosensitivity on clinical assessment of a patient and that there are usually clues in the patient's history and examination findings. It is also advisable to have a low threshold when referring for investigation, for example in a patient with longstanding atopic dermatitis in whom there may be a history of change in the seasonal pattern of their disease. They may have problems in the summer more than in the winter or they may have sites of involvement such as on the head and neck for the first time and it may well be that this patient has developed chronic actinic dermatitis. In this setting, particularly if the narrowband UVB MED that is undertaken prior to phototherapy is abnormal, the patient should be referred for monochromator phototesting to exclude chronic actinic dermatitis. A range of investigations is available - not all of these will be used in all patients but will be adapted according to the clinical presentation, for example druginduced photosensitivity, lupus or cutaneous porphyrias.

The longer term risks of chronic photosensitivity are not well understood although in the idiopathic photodermatoses there is no evidence of any increased risk of skin cancer or other malignancies. Indeed, in polymorphic light eruption there appears to be a reduced lifetime risk of skin cancer even allowing for the confounding factor of photoprotection. ${ }^{12}$ The association between drug phototoxicity and possible skin cancer risk is becoming clearer with the use of some drugs which seem to be associated with an increased risk of skin cancer, such as the phototoxic fluoroquinolones, voriconazole and the BRAF inhibitors, notably vemurafenib. The mechanisms underlying any link between drug phototoxicity and photocarcinogenesis, or potential ocular or systemic toxicity, are not fully clarified and this area requires further investigation. ${ }^{13}$

\section{REFERENCES}

I Fitzpatrick TB. Ultraviolet-induced pigmentary changes: benefits and hazards. Curr Probl Derm 1986; 15: 25-38.

2 Kerr AC, Muller F, Ferguson J et al., Occupational carprofen photoallergic contact dermatitis. Br J Dermatol 2008; 159: 1303-8. http://dx.doi.org/I 0.III I/j.1365-2/33.2008.08847.x

3 Stafford R, Farrar MD, Kift R et al. The impact of photosensitivity disorders on aspects of lifestyle. $\mathrm{Br} J$ Dermatol 2010; 163: 817-22. http://dx.doi.org/I0.IIII/j.1365-2133.2010.09905.x

4 Jong CT, Finlay AY, Pearse AD et al. The quality of life of 790 patients with photodermatoses. Br J Dermatol 2008; 159: 192-7. http://dx.doi.org/I 0.II I I/j.1365-2I33.2008.0858I.x

5 Richards HL, Ling TC, Evangelou G et al. Evidence of high levels of anxiety and depression in polymorphic light eruption and their association with clinical and demographic variables. $\mathrm{Br} J$ Dermatol 2008;159:439-44.http://dx.doi.org//0.IIII/j.1365-2I33.2008.08623.x

6 Rizwan M, Reddick CL, Bundy C et al. Photodermatoses: environmentally induced conditions with high psychological impact. Photochem Photobiol Sci 2013; 12: 182-9. http://dx.doi.org/10.1039/ c2pp25177a

7 The European Multicentre Photopatch Test Study (EMCPPTS) Taskforce. A European multicentre photopatch test study. $\mathrm{Br} J$ Dermatol 2012; 166: 1002-9. http:dx.doi. org/I0.IIII/j.1365-2I33.20I2.10857.x

8 Fenton L, Dawe R, Ibbotson S et al. Impact assessment of energyefficient lighting in patients with lupus erythematosus: a pilot study. Br J Dermatol 20 I4; I70:694-8. http://dx.doi.org/ I0.1 I I I/bjd. $127 / 9$

9 Moseley $\mathrm{H}$, Ferguson J. The risk to normal and photosensitive individuals from exposure to light from compact fluorescent lamps. Photodermatol Photoimmunol Photomed 201 I; 27: 131-7. http://dx. doi.org/I0.1III/j.1600-078I.20II.00576.x

10 Fenton L, Ferguson J, lbbotson $\mathrm{S}$ et al. Energy saving lamps and their impact on photosensitive and normal individuals. $\mathrm{Br} J$ Dermatol 2013; 169: 910-5. http://dx.doi.org/I0.1 I I I/bjd.12457

I I Rhodes LE, Bock M, Janssens AS et al. Polymorphic light eruption occurs in $18 \%$ of Europeans and does not show higher prevalence with increasing latitude: multicenter survey of 6,895 individuals residing from the Mediterranean to Scandinavia. J Invest Dermatol 2010; 130: 626-8. http://dx.doi.org// 0.1038/jid.2009.250

12 Lembo S, Fallon J, O'Kelly $P$ et al. Polymorphic light eruption and skin cancer prevalence: is one protective against the other? $\mathrm{Br} J$ Dermatol 2008; 159: 1342-7. http://dx.doi. org/I 0.1 III/j. I365-2/33.2008.08734.x

13 O'Gorman SM, Murphy GM. Photosensitizing medications and photocarcinogenesis. Photodermatol Photoimmunol Photomed 2014; 30: 8-14. http://dx.doi.org//0.1 III/phpp. 12085 